Pacific Northwest

National Laboratory

Operated by Battelle for the

U.S. Department of Energy

\title{
Preventing Buoyant Displacement Gas Release Events in Hanford Double-Shell Waste Tanks
}

\author{
P. A. Meyer \\ C. W. Stewart
}

January 2001

Prepared for the U.S. Department of Energy under Contract DE-AC06-76RL01830 


\title{
DISCLAIMER
}

This report was prepared as an account of work sponsored by an agency of the United States Government. Neither the United States Government nor any agency thereof, nor Battelle Memorial Institute nor any of their employees makes any warranty, express or implied, or assumes any legal liability or responsibility for the accuracy, completeness, or usefulness of any information, apparatus, product, or process disclosed or represents that its use would not infringe privately owned rights. Reference herein to any specific commercial product, process, or service by trade name, trademark, manufacturer, or otherwise does not necessarily constitute or imply its endorsement, recommendation, or favoring by the United States Government or any agency thereof, or Battelle Memorial Institute. The views and opinions of authors expressed herein do not necessarily state or reflect those of the United States Government or any agency thereof.

\section{PACIFIC NORTHWEST NATIONAL LABORATORY operated by \\ BATTELLE \\ for the \\ UNITED STATES DEPARTMENT OF ENERGY under Contract DE-AC06-76RL01830}

\author{
Printed in the United States of America \\ Available to DOE and DOE contractors from the \\ Office of Scientific and Technical Information, \\ P.O. Box 62, Oak Ridge, TN 37831-0062; \\ ph: (865) 576-8401 \\ fax: (865) 576-5728 \\ email: reports@adonis.osti.gov \\ Available to the public from the National Technical Information Service, \\ U.S. Department of Commerce, 5285 Port Royal Rd., Springfield, VA 22161 \\ ph: (800) 553-6847 \\ fax: (703) 605-6900 \\ email: orders@ntis.fedworld.gov \\ online ordering: http://www.ntis.gov/ordering.htm
}




\title{
Preventing Buoyant Displacement Gas Release Events in Hanford Double-Shell Waste Tanks
}

\author{
P. A. Meyer
}

C. W. Stewart

January 2001

Prepared for the U.S. Department of Energy under Contract DE-AC06-76RLO 1830

Pacific Northwest National Laboratory

Richland, Washington 99352 


\section{Summary}

The report summarizes the predictive methods used to ensure that waste transfer operations in Hanford waste tanks do not create waste configurations that lead to hazardous gas retention and sudden gas release events (GREs). The gas release behavior of the waste in existing doubleshell tanks (DSTs) has been reasonably well characterized, and the flammable gas safety issues associated with safe storage of waste are being formally resolved. However, waste is also being transferred between DSTs and from single-shell tanks (SSTs) into DSTs by salt-well pumping and sluicing, which creates new wastes and waste configurations that have not been characterized. Additionally, planning is under way for various waste transfer scenarios to support waste feed delivery to the proposed vitrification plant. It is critical that such waste transfers do not create waste conditions with the potential for dangerous GREs.

The basic mechanism for large, spontaneous GREs is referred to as buoyant displacement. The physics of a buoyant displacement GRE (BDGRE) is reviewed, and the theories of buoyant bodies and gas accumulation are summarized in this report. The physical parameters important for determining gas retention characteristics are presented. The fill histories leading to problematic tanks are discussed, as is the potential for creating new tanks that exhibit BDGREs. The conditions that exacerbate gas retention and increase the probability of BDGRE behavior are

- deep convective layer (more than one to two meters)

- deep nonconvective layer (more than one to two meters)

- low neutral buoyancy void fraction (small difference between convective and nonconvective layer densities)

- high gas generation rate.

The parametric indicators used to identify tanks that exhibit BDGREs are presented. The basis of these indicators is either empirical, chemical, or physical. The origins of the indicators are reviewed and their physical bases are discussed.

Also discussed are issues of concern in applying the parametric indicators when waste is transferred between tanks or altered in-tank. The discussion includes potential changes in waste configuration that affect BDGRE behavior and what must be considered in predicting the properties of a future tank. Examples of the SY-101 transfer and dilution and the C-106 to AY-102 sluicing projects are given. 


\section{Contents}

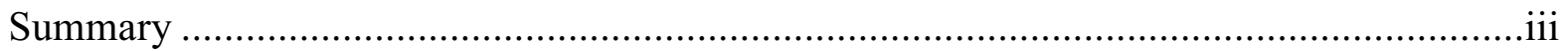

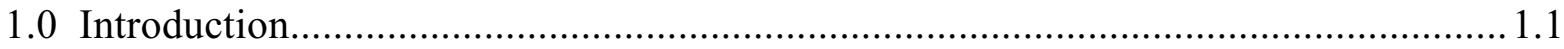

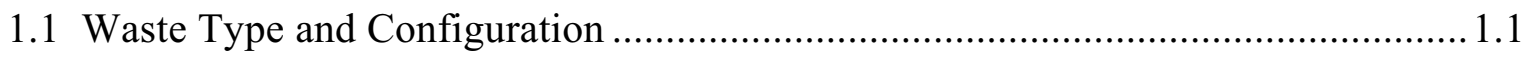

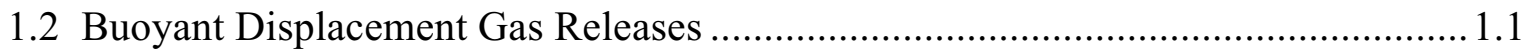

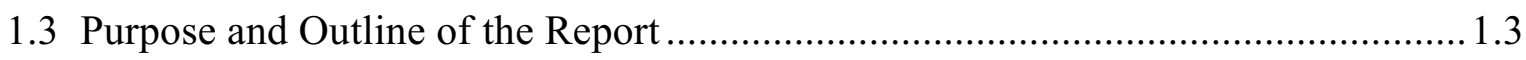

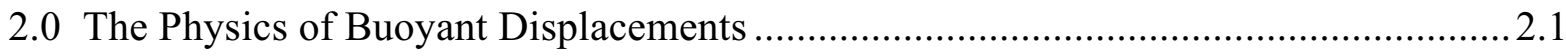

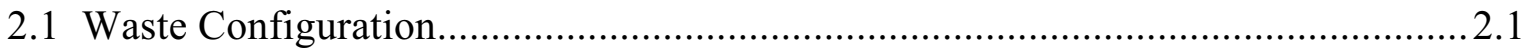

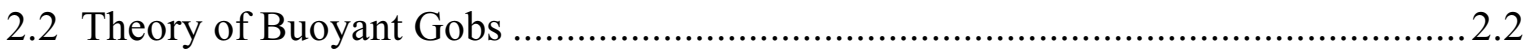

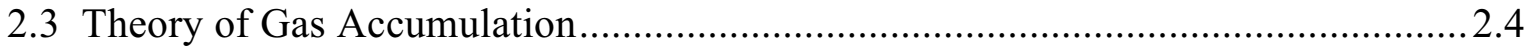

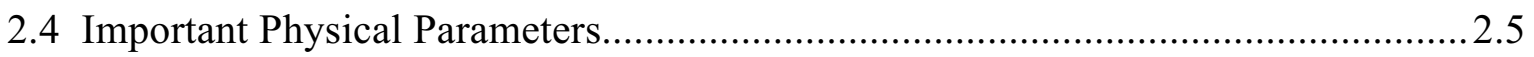

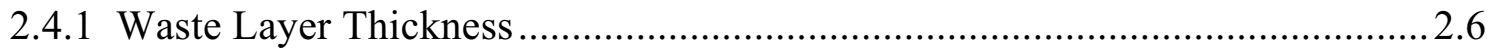

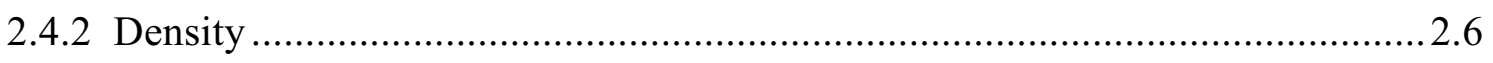

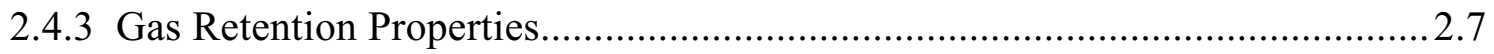

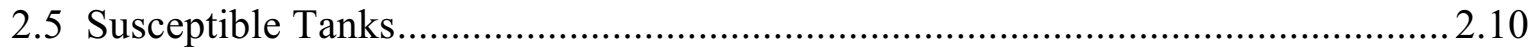

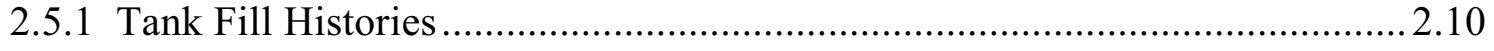

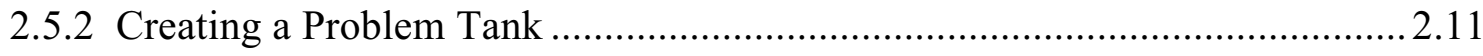

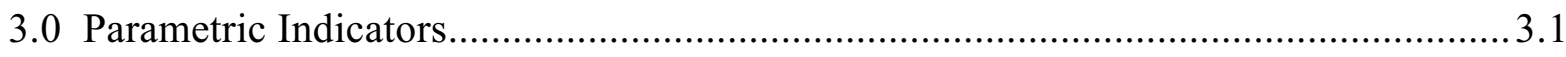

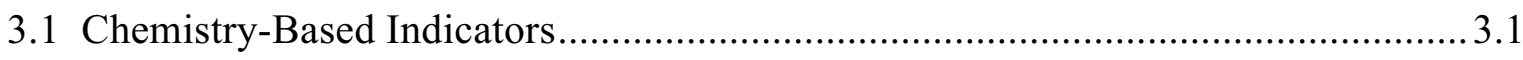

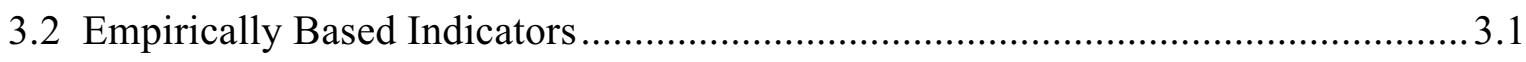

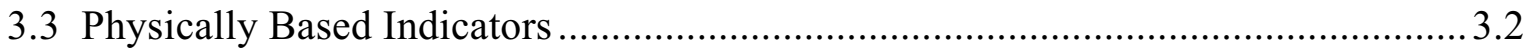

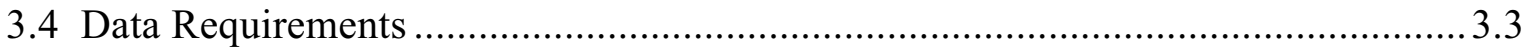

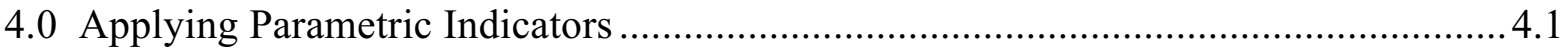

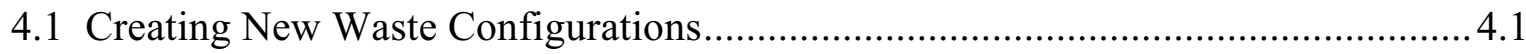

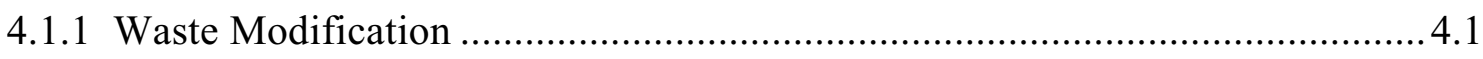

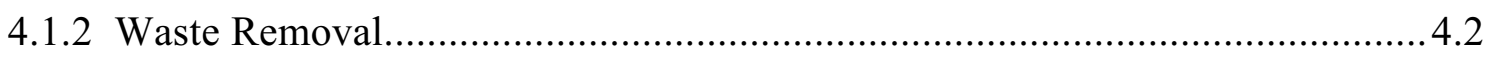

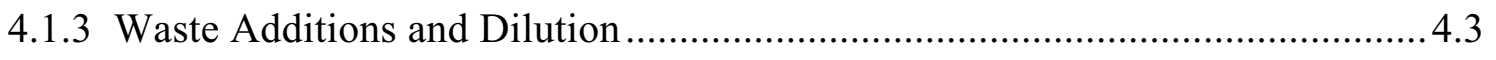

4.2 Important Issues in Predicting the Waste Configuration............................................ 4.3

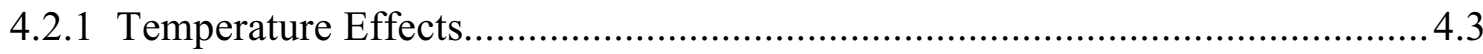

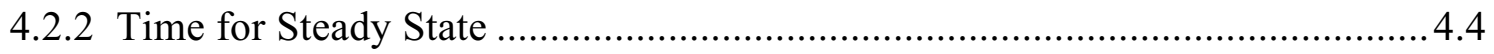

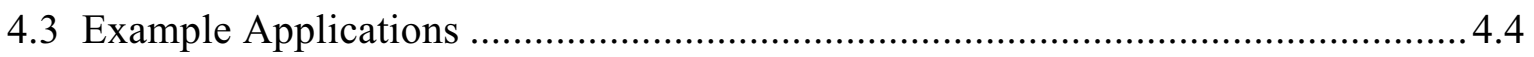

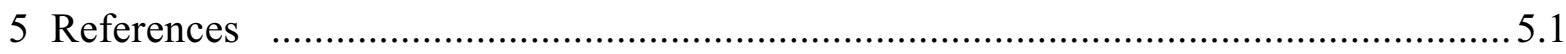




\section{Figures}

1.1 Waste Configuration Susceptible to Buoyant Displacement Gas Release Events .............. 1.2

2.1 The BDGRE Process in Hanford DSTs ..................................................................2.2

4.1 Buoyancy Ratio Parameter Values for all Hanford Double-Shell Tanks ......................... 4.5

4.2 $\mathrm{SpG}_{\mathrm{CL}} * \mathrm{H}_{\mathrm{NCL}}$ Parameter Values for all Hanford Double-Shell Tanks ............................ 4.6

4.3 Average Specific Gravity for all Hanford Double-Shell Tanks ..................................... 4.6

\section{Tables}

2.1 SY-101 Fill Summary 1977 Through 1980........................................................2.11

3.1 Inputs Required for Parametric Indicators .............................................................. 3.4 


\subsection{Introduction}

Approximately $200,000 \mathrm{~m}^{3}$ (53 million gallons) of radioactive waste is stored in Hanford waste tanks (Hanlon 2000). The waste is stored in 177 carbon steel tanks with capacities of 190 to $4500 \mathrm{~m}^{3}$ (50 to $1200 \mathrm{kgal}$ ). Of these, 149 are single-shell tanks (SSTs) built in the 1940s, 1950s, and early 1960s, and 28 are newer double-shell tanks (DSTs) constructed in the 1970s. The SSTs are no longer being used, and their waste is being transferred to the DSTs, which will remain in service for many more years. The behavior of the waste stored in the DSTs is the focus of this report.

\subsection{Waste Type and Configuration}

The waste in these tanks is broadly classified as saltcake, sludge, and supernate. Saltcake consists of sodium salts that precipitated from saturated liquid on cooling. Sludge consists of fine insoluble particles that are mostly metal oxides. Some tanks contain a mixture of sludge and saltcake; however, the mixed waste types appear to behave like saltcake. Supernate is simply the liquid left after the solids settle out. The supernate associated with saltcake contains dissolved salts and thus has a high density. The supernate over sludge is less dense because the sludge solids species are much less soluble.

The aging SSTs were removed from active use in 1980 because of concerns about their physical integrity. Since then, much of their pumpable liquid has been transferred to DSTs to reduce the potential for leaks. The liquid transferred to the DSTs was typically concentrated by evaporation. When saltcake waste cooled after evaporation, it precipitated relatively deep layers of sodium salt particles. Other DSTs received high-activity sludge with a very high heat load and gas generation rate. But the sludge settled into much shallower layers than the saltcake did.

In either waste type, these processes have formed a waste configuration consisting of a layer of supernatant liquid overlying a sediment layer of settled solids. Because the liquid is mixed by natural convection, it is called the convective layer. The strength of the sediment inhibits convection so it is called the nonconvective layer. In some tanks, there is also a floating layer of solids and trapped gas bubbles that is termed the "crust." This waste configuration is shown schematically in Figure 1.1.

\subsection{Buoyant Displacement Gas Releases}

All radioactive Hanford wastes generate a flammable gas mixture consisting mainly of hydrogen (fuel), nitrous oxide (oxidizer), nitrogen (inert), and small amounts of ammonia, methane, and other hydrocarbons. These gases are generated by both radiolysis of water and thermal and radiolytic decomposition of organic solvents. Some tanks have stored a relatively large volume of this gas, creating the potential for a sudden gas release into the tank headspace. A sufficiently large release could make the tank headspace flammable for a few hours or days until it is dissipated by ventilation. If the flammable mixture in the headspace were to be ignited, the resulting pressure might fail the tank and release radioactive material to the environment. 


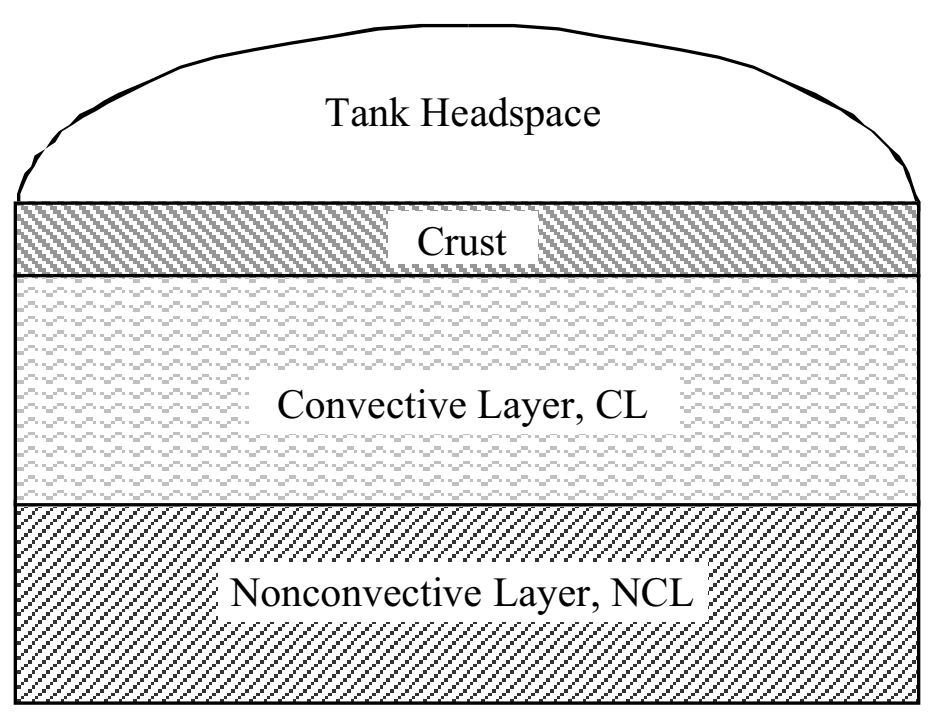

Figure 1.1. Waste Configuration Susceptible to Buoyant Displacement Gas Release Events

The flammable gas hazard in Hanford waste tanks was first recognized in the behavior of DST 241-SY-101 (SY-101). The waste level in this tank began periodically rising and suddenly dropping shortly after it was filled in 1980. The large, sawtooth level drops were taken as an indication of gas release events (GREs) that might pose a safety hazard. Intense study of this tank's behavior during 1990 to 1992 revealed that these releases were, in fact, hazardous; the gas was indeed flammable, and the releases were quite large. Some were of sufficient volume to exceed the lower flammability limit (LFL) in the tank headspace and might have damaged the tank had the gas been ignited. The major concern in SY-101 was mitigated in late 1993 with the installation of a mixer pump that prevented gas retention in the nonconvective layer (Allemann et al. 1994; Stewart et al. 1994; Brewster et al. 1995).

The historic gas releases in SY-101 prior to mixing are now understood to be buoyancyinduced displacement events, at one time called "rollovers" (Allemann et al. 1993). In a buoyant displacement gas release event (BDGRE), a portion, or "gob," of the nonconvective layer accumulates gas until it becomes sufficiently buoyant to overcome the weight and strength of material restraining it. At that point, it suddenly breaks away and rises through the convective layer. The trapped gas bubbles expand as the gob rises, failing the surrounding material, so a portion of the gas can escape from the gob into the headspace. After releasing a portion of its gas, the remainder of the gob loses buoyancy and sinks back to the nonconvective layer.

Theory, experiment, and experience indicate that only the liquid-over-solid waste configuration found in the DSTs has the potential for significant gas releases by buoyant displacement. Only SY-103, AW-101, AN-103, AN-104, and AN-105 now actually exhibit this kind of gas release. However, since $1995,80 \%$ of these releases have been less than 10 cubic meters in volume, compared with over 100 cubic meters in a typical SY-101 release. In terms of the flammability limit, these releases are quite small, with only the largest ones slightly exceeding $25 \%$ of the lower flammability limit (LFL) just twice in one tank since 1995. The release volumes have also been decreasing. From 1995 through 1996, 30\% of the releases were 5 cubic meters or 
less. From 1997 through 1999, 76\% were in this range. Nevertheless, it is undesirable to create this behavior in any more tanks.

\subsection{Purpose and Outline of the Report}

The gas release behavior of the waste in the existing DSTs has been reasonably well characterized (Hedengren et al. 2000), and the flammable gas safety issues associated with storing waste in the current configuration are now being formally resolved. However, waste is also being transferred between DSTs and removed from SSTs into DSTs, creating new wastes and waste configurations that are potentially different from those that have already been characterized. These operations have supported remediation of problematic tanks, including sluicing of high-heat waste from SST C-106 to the DST AY-102 and transfer and back-dilution campaigns in SY-101. In each of these cases, no BDGRE waste configuration was created. The benign waste behavior in these tanks following the operations helps to validate the predictive methods used.

Planning is under way for various waste transfer scenarios to support waste feed delivery to the proposed vitrification plant. It is critical that such waste transfers do not create waste conditions with the potential for BDGREs. Based on the positive results with AY-102 and SY-101, we recommend using the current BDGRE prediction methods to evaluate and plan these future operations. The purposes of this report are to summarize those methods and describe how they should be used.

The physics of BDGREs is reviewed in Section 2. The theories of buoyant gobs and gas accumulation are summarized. The role of waste configuration is addressed, and the important physical parameters affecting BDGRE behavior are discussed. Additionally, the fill history in the problematic tanks is addressed. Finally, the potential for creating new tanks that exhibit BDGREs is summarized.

In Section 3, parametric indicators are presented that have been used successfully to identify tanks that exhibit BDGREs. The origins of the indicators are reviewed and their physical bases discussed.

Section 4 addresses the issues of concern to BDGRE behavior when waste is transferred between tanks or altered in-tank. The discussion includes potential changes in waste configuration that affect BDGRE behavior. The section also includes a discussion of what must be considered in predicting the properties of a future tank. Examples of the SY-101 transfer and dilution and the C-106 to AY-102 sluicing projects are given. Section 5 lists the references cited in the report. 


\subsection{The Physics of Buoyant Displacements}

Buoyant displacement gas releases are dominated by relatively simple mechanisms that follow basic physical principles. Waste subject to buoyant displacements should not be viewed as a "virus" of which a small volume can "infect" a receiver tank and cause bad behavior there. It is the physical properties and configuration of the waste, not its precise chemical composition, that controls whether this gas release mechanism occurs. This section discusses the important factors inherent in buoyant displacements. The influence of waste configuration and waste type is described in Section 2.1. The theory of buoyant gobs is summarized in Section 2.2, and the theory of gas accumulation is presented in Section 2.3. Section 2.4 focuses on the most important physical parameters that determine whether buoyant displacements are likely, and Section 2.5 gives examples of which tanks are susceptible to BDGREs.

\subsection{Waste Configuration}

As discussed in Section 1.1, BDGREs have been observed only in saltcake DSTs with supernate overlying a deep nonconvective layer at the bottom of the tank. Most of the characterization effort has focused on saltcake waste. No measurements have been made of retained gas in sludge tanks; however, the physical characteristics of sludge waste are postulated to be similar with respect to potential BDGREs. In either waste, the nonconvective layer is typically a weak pseudo-plastic solid with a consistency ranging from that of a thin milk shake (a yield strength of around $10 \mathrm{~Pa}$ ) at the top to that of sticky mud or wet clay (200-300 Pa) near the bottom. The strength increases roughly linearly with depth (Hedengren et al. 2000). When the nonconvective layer material is mobilized (by a mixer pump jet, mechanical disturbance, or other such means), it behaves as a highly shear-thinning viscous fluid with viscosity on the order of thousands of centipoise at low shear rates. The density of a nonconvective layer is generally in the range of $1300-1800 \mathrm{~kg} / \mathrm{m}^{3}$. Because the nonconvective layer possesses a degree of mechanical strength, it is possible for gas bubbles generated within it to accumulate.

The convective layer has no mechanical strength and can be adequately described as a Newtonian liquid. The viscosity of the convective layer is typically in the $1-10 \mathrm{cP}$ range (Stewart et al. 1996). The density of the convective layer depends on the mass of dissolved soluble solids, but it is generally in the $1100-1300 \mathrm{~kg} / \mathrm{m}^{3}$ range above the relatively insoluble sludge and 1300-1500 kg/m $\mathrm{m}^{3}$ above soluble saltcake. Given that the convective layer has no mechanical strength, gas is not retained there, although a very small volume of rising gas bubbles is typically present.

In addition to the convective and nonconvective layers, most BDGRE tanks have a floating crust layer on top of the convective layer. This crust layer is believed to be maintained by a combination of processes involving transport of solids to the surface of the convective layer by rising bubbles, precipitation at the surface due to cooling, and accumulation of some remnants of BDGREs. The crust layer, while common to BDGRE tanks, plays no active role in the BDGRE process. 


\subsection{Theory of Buoyant Gobs}

In a buoyant displacement, a portion, or "gob," of the nonconvective layer accumulates gas until it becomes sufficiently buoyant to overcome its weight and the strength of the surrounding material restraining it. At that point it breaks away and rises through the convective layer. The stored gas bubbles expand as the gob rises, failing the surrounding material, so a portion of the gas can escape from the gob into the headspace. After releasing a portion of its gas, the remaining gob material is no longer buoyant and sinks back to the bottom of the tank. This gas release process defines the BD GRE. The buoyant displacement gas release process is illustrated in Figure 2.1. Note that the gobs are staggered for clarity only: the actual process is believed to occur more or less vertically. The basic theory of buoyant gobs assumes uniform waste properties. We know that actual waste properties vary to some extent in the tanks. However, because the basic phenomenon of interest occurs on a large scale, the presence of small-scale variations can be ignored when considering first-order dominant behavior. Nevertheless, if the waste is significantly nonuniform, the model may or may not apply.

The retained gas volume required for buoyancy is determined by the ratio of the bulk densities of the convective and nonconvective layers. ${ }^{(a)}$ The gas volume fraction or void fraction in the nonconvective layer at neutral buoyancy (bulk density of nonconvective layer equal to the convective layer density) is given by

$$
\alpha_{\mathrm{NB}}=1-\frac{\rho_{\mathrm{CL}}}{\rho_{\mathrm{NCL}}}
$$

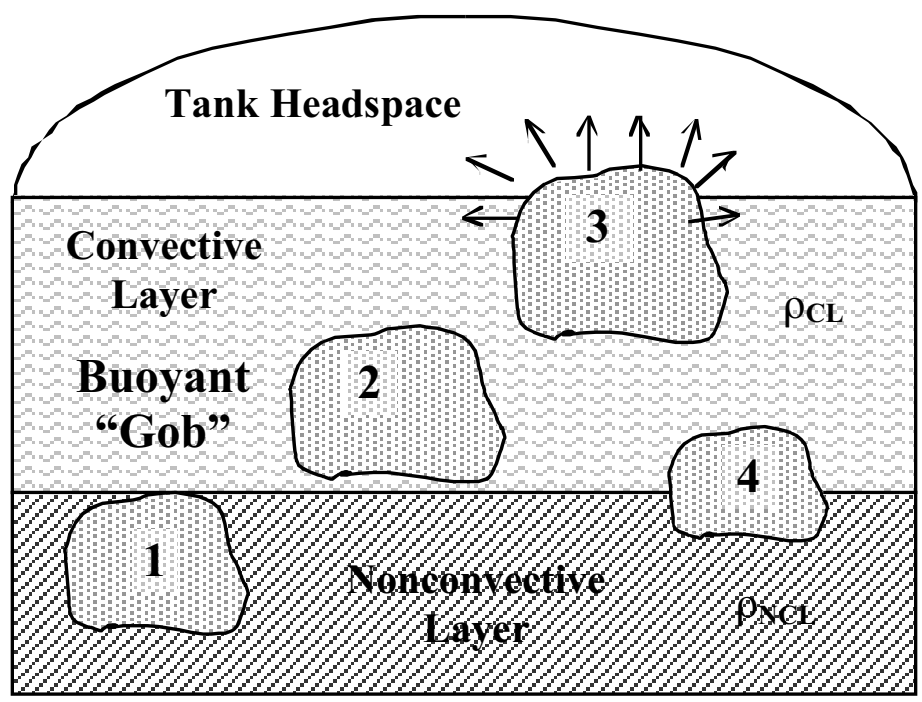

Figure 2.1. The BDGRE Process in Hanford DSTs: the gob 1) becomes buoyant, 2) breaks free of nonconvective layer, 3) expands, releasing gas, and

4) sinks back into nonconvective layer (likely breaks up during process)

(a) A BDGRE requires a gas volume higher than neutral buoyancy to counteract the restraining effect of waste strength. However, the effect is small due to the low strength of DST nonconvective layers and low surface-to-volume ratio of large gobs. Eq. (2.1) gives an accurate first-order representation. 
where $\rho_{\mathrm{CL}}$ and $\rho_{\mathrm{NCL}}$ are the bulk degassed densities of the convective and nonconvective layers, respectively. ${ }^{\text {(a) }}$ In concentrated saltcake waste, where the convective and nonconvective layer densities are similar, a relatively small gas volume is required for buoyancy.

Individual gobs are believed to reach buoyancy and undergo buoyant displacement more or less independently, so different regions of the tank are in different stages of evolution toward the event with a timing that becomes almost random. The effect is similar to popcorn, with each grain heating up and popping independently of the rest.

This concept of independent gobs is consistent with observed GRE patterns. In-tank video during large gas releases in SY-101 clearly shows a series of local upwellings from different areas of the tank. Waste temperature profiles before and after GREs observed in other DSTs show the effects of very few of them. Based on actual gas release volumes, the nonconvective layer depth, and the neutral buoyancy void fraction, a maximum of $10 \%$ of the nonconvective layer must have become buoyant to produce each GRE. Finally, this concept describes the observed GRE period. Tank AN-105, for example has a typical GRE interval of 270 days after 1993, and the period is highly variable. Based on the observed gas retention rate deduced from waste level rise between events, the GRE period should be about six years. However, if the number of randomly evolving gobs is about eight, the frequency of GREs is brought into line with observation. This same argument applies in the other BDGRE tanks.

This model suggests that, eventually, random buoyant displacement events involving gobs 8 to $10 \mathrm{~m}$ in diameter will occur in the existing DSTs. Thus, 5 to 10 gobs will be in random stages of GRE development in each tank. Variability in GRE size and period comes from both randomness of the evolution stage of the gobs and the specific condition left by the preceding GRE of a specific gob.

Some of the larger releases require participation of multiple gobs. In SY-101, the video shows that large gas releases involved four or five gobs of various sizes. In the other tanks, there are several examples of closely spaced double peaks in the headspace hydrogen concentration indicating that at least two gobs have participated. Though the basic model assumes the gobs behave independently, the buoyant displacement process obviously causes a relatively strong disturbance. If another gob close by the first one is buoyant but still restrained by the strength of the surrounding waste, this disturbance might be enough to break it free.

It is postulated that the transition from single- to multiple-gob buoyant displacements is influenced by the gas generation rate. A higher gas generation rate would cause a greater number of gobs to approach buoyancy at the same time, thereby increasing the probability that more than one could be released simultaneously. Evidence for this may be seen in the decreased GRE volume in the five BDGRE DSTs in the last several years. This change is coincident with a $5-10^{\circ} \mathrm{F}$ waste temperature decrease, which has reduced the gas generation rate by as much as $50 \%$ (Hedengren et al. 2000).

(a) Theoretically, the convective layer density used in Eq. (2.1) should account for any gas which may be present in the convective layer. The convective layer, while not retaining gas by its strength, can contain gas due to rising bubbles. However, the amount of gas will be small, and the effect on the convective layer density will be negligible. 


\subsection{Theory of Gas Accumulation}

Why do some tanks exhibit BDGREs, yet others do not? Most tanks apparently achieve a steady state where gas generation is balanced by a steady background release, so the neutral buoyant void fraction given by Eq. (2.1) is never attained. In a few tanks, however, the background release remains less than the gas generation rate, allowing gas to accumulate to the point of buoyancy. To explain the phenomenon, mass conservation on the gas in the nonconvective layer is expressed mathematically in the following integral balance:

$$
\frac{\mathrm{d}}{\mathrm{dt}} \int_{\mathrm{V}} \mathrm{mdV}+\int_{\mathrm{A}} \mathrm{J}_{\mathrm{m}} \mathrm{dA}=\int_{\mathrm{V}} \mathrm{gdV}
$$

The first term in Eq. (2.2) is the rate of gas accumulation, the second term represents the loss of gas through steady release, and the last term is the gas generation rate. The mass $\mathrm{m}$ is expressed as the number of moles of gas per unit total volume of waste, $J_{\mathrm{m}}$ is the flux of gas across the surface of the nonconvective layer (moles $\left./ \mathrm{m}^{2}-\mathrm{s}\right)$ and $\mathrm{g}$ is the gas generation rate (moles $\left./ \mathrm{m}^{3}-\mathrm{s}\right)$. The nonconvective layer volume is $\mathrm{V}\left(\mathrm{m}^{3}\right)$ and its upper surface area is A $\left(\mathrm{m}^{2}\right)$. The volume and surface area are related by the height of the nonconvective layer, $\mathrm{V}=\mathrm{A} \mathrm{H}_{\mathrm{NCL}}$.

Using the ideal gas law, $\mathrm{m}$ and $\mathrm{J}_{\mathrm{m}}$ can be expressed in terms of volume so that Eq. (2.2) becomes

$$
\frac{\mathrm{d}}{\mathrm{dt}} \int_{\mathrm{V}} \alpha \frac{\mathrm{p}}{\mathrm{RT}} \mathrm{dV}+\int_{\mathrm{A}} \mathrm{J}_{\alpha} \frac{\mathrm{p}}{\mathrm{RT}} \mathrm{dA}=\int_{\mathrm{V}} \mathrm{gdV}
$$

Here, $\alpha$ is the local gas volume fraction in the nonconvective layer; $\mathrm{p}$ and $\mathrm{T}$ are the local pressure $(\mathrm{Pa})$ and temperature $(\mathrm{K})$, respectively; $\mathrm{R}$ is the gas constant $(\mathrm{J} / \mathrm{mole}-\mathrm{K})$; and $\mathrm{J}_{\alpha}$ is the gas volume flux across the nonconvective layer surface $\left(\mathrm{m}^{3} / \mathrm{m}^{2}-\mathrm{s}\right)$. Applying appropriate averaging and assuming the nonconvective layer pressure and temperature are constant yield the following expression of the gas retention rate:

$$
\frac{\mathrm{d} \alpha}{\mathrm{dt}}=\mathrm{g} \frac{\mathrm{RT}}{\mathrm{p}}-\frac{\mathrm{J}_{\alpha}}{\mathrm{H}_{\mathrm{NCL}}}
$$

In Eq. (2.4), $\alpha, p$, and $\mathrm{T}$ are now the average gas fraction, pressure, and temperature in the nonconvective layer, respectively, and $\mathrm{J}$ is the average gas volume flux leaving its upper surface.

The exiting volume flux can be described by several mechanisms. If the gas in the nonconvective layer is stored as connected dendritic bubbles, the gas might escape by flowing through the connected passages. In the much more likely scenario that the gas accumulates as individual bubbles, the flux is represented by the number density, size, and rise velocity of bubbles crossing the upper surface of the nonconvective layer or simply as the bubble number flux and size. These options are given symbolically by

$$
\mathrm{J}_{\alpha}=\alpha^{*} \mathrm{u}^{*}=\mathrm{n}^{*} \mathrm{~V}_{\mathrm{b}}^{*} \mathrm{u}_{\mathrm{b}}^{*}=\mathrm{k}^{*} \mathrm{~V}_{\mathrm{b}}^{*}
$$


where $\alpha^{*}$ and $u^{*}$ are the gas fraction and velocity $(\mathrm{m} / \mathrm{s})$ at the upper surface of the nonconvective layer, respectively. The symbols $\mathrm{n}^{*}, \mathrm{~V}_{\mathrm{b}}{ }^{*}$, and $\mathrm{u}_{\mathrm{b}}{ }^{*}$ are the bubble number density (\#bubbles $/ \mathrm{m}^{3}$ ), average bubble volume $\left(\mathrm{m}^{3} / \mathrm{bubble}\right)$, and the bubble rise velocity $(\mathrm{m} / \mathrm{s})$ at the upper surface of the nonconvective layer; and $\mathrm{w}^{*}$ is the bubble number flux (\#bubbles $/ \mathrm{m}^{2}-\mathrm{s}$ ) crossing the upper surface of the nonconvective layer.

The volumetric generation rate decreases and the nonconvective layer depth increases in direct proportion to an increase in void fraction. If $\mathrm{g}_{0}$ and $\mathrm{H}_{0}$ are the values of the gas generation rate and nonconvective layer depth at zero void fraction, then $g=\mathrm{g}_{0}(1-\alpha)$ and $\mathrm{H}_{\mathrm{NCL}}=\mathrm{H}_{0} /(1-\alpha)$. Substituting these relations and Eq. (2.5) into Eq. (2.4) yields

$$
\frac{1}{1-\alpha} \frac{\mathrm{d} \alpha}{\mathrm{dt}}=\mathrm{g}_{0} \frac{\mathrm{RT}}{\mathrm{p}}-\frac{\mathrm{n}^{*} \mathrm{~V}_{\mathrm{b}}^{*} \mathrm{u}_{\mathrm{b}}^{*}}{\mathrm{H}_{0}}
$$

In Eq. (2.6), the gas generation term is essentially unaffected by gas accumulation. The gas release term, however, tends to increase with the void fraction. As gas accumulates in the nonconvective layer, the bubble number density, the bubble size, or both must increase. If the bubble rise velocity is assumed to depend on bubble size, it will also increase. The net effect is that the gas release rate term increases in more than direct proportion to gas accumulation.

Eq. (2.6) shows that, as long as the generation term is larger than the release term, the void fraction will increase with time. If this process continues, the void fraction increases to neutral buoyancy, initiating a BDGRE. However, since the release term also increases with gas accumulation, generation and release will eventually be equal. If the average void fraction remains below the neutral buoyant fraction at this point, then a BDGRE is not possible.

\subsection{Important Physical Parameters}

The basic theory discussed in the preceding sections suggests that certain physical parameters associated with DST waste play a key role in determining whether a tank will exhibit BDGREs. Equation (2.6) shows that, if the pressure is reduced or the temperature is increased, each mole of gas created occupies a proportionally greater volume, which increases the gas generation term. The temperature also has a very powerful effect on the rates of the chemical reactions responsible for the molar gas generation rate, g. Besides temperature, the molar generation rate depends on the chemical composition of the waste, radioactivity, and liquid fraction, as is discussed in Section 4.

The magnitude of the gas release term depends on several things. First, it is important to note that the nonconvective layer height in the denominator of the last term in Eq. (2.6) is an artifact of geometry, not of any specific model or assumption of the gas generation or release mechanism. This means that a shallow nonconvective layer will have twice the release rate of one twice as deep (all else being equal). The bubble number density, velocity, and maximum bubble size all depend on the "gas retention properties" of the nonconvective layer material. This is a rather imprecise class of attributes that includes the bulk material strength, particle size, shape, and interstitial pore size relative to the bubble size, bubble interface contact angle with the particles, degree of particle compaction, interparticle forces, etc. Assuming creeping flow, the 
bubble velocity also depends on bubble size and shape and the apparent nonconvective layer viscosity. The role of these and other parameters in relation to BDGRE behavior is summarized in this section.

\subsubsection{Waste Layer Thickness}

The thicknesses of the convective and nonconvective layers are important parameters affecting BDGRE behavior. The thickness of the nonconvective layer is important for several independent but related reasons. First, according to Eq. (2.6), the thicker the layer the lower the release rate relative to generation, resulting in an increased void growth rate. Also, results of bubble transport modeling in nonconvective layers (Meyer and Wells 2000) show the average void fraction in the nonconvective layer increases approximately as the square of $\mathrm{H}_{\mathrm{NCL}}$. Finally, the total releasable volume of gas stored in the nonconvective layer is directly related to $\mathrm{H}_{\mathrm{NCL}}$. Given that void growth rate, average void fraction, and total gas volume all increase with $\mathrm{H}_{\mathrm{NCL}}$, we conclude that the potential for BDGREs and the hazard they represent increases nonlinearly with nonconvective layer thickness. Conversely, tanks with a shallow nonconvective layer have minimal BDGRE risk.

The thickness of the convective layer is also an important factor. First of all, for buoyant displacement to occur, a convective layer at least a meter or two in depth must be present. If the convective layer is very shallow, a gob may become buoyant but cannot rise enough for the expanding gas to fail the surrounding material. Hence, removing the supernatant is an effective preventive for BDGREs. On the other hand, if the convective layer is quite deep, a buoyant gob releases a large amount of potential energy that breaks it up and releases its gas as it rises. The analysis of buoyant gobs in terms of available energy was derived by Meyer et al. (1997) and further refined by Meyer and Wells (2000).

Additionally, the total waste depth $\left(\mathrm{H}_{\mathrm{NCL}}+\mathrm{H}_{\mathrm{CL}}+\right.$ crust thickness) determines the average pressure at which gas is stored. All other conditions being equal, when the total depth is increased, the average gas pressure in the nonconvective layer increases linearly with depth. Hence, the volume of gas potentially releasable in the dome headspace also increases with waste depth. Further, because the headspace decreases as the waste depth increases, the volume of gas release necessary to make the headspace atmosphere flammable decreases as well.

\subsubsection{Density}

The ratio of densities of the convective and nonconvective layers determines the neutral buoyancy void fraction, $\alpha_{\mathrm{NB}}$, via Eq. (2.1). The convective layer density, $\rho_{\mathrm{CL}}$, is determined primarily by the mass of soluble solids dissolved in the liquid. The convective layer density ranges from $1100 \mathrm{~kg} / \mathrm{m}^{3}$ (dilute) to $1300 \mathrm{~kg} / \mathrm{m}^{3}$ (some soluble solids present) in sludge tanks and from $1300 \mathrm{~kg} / \mathrm{m}^{3}$ (dilute) to $1500 \mathrm{~kg} / \mathrm{m}^{3}$ (highly concentrated) in saltcake. The nonconvective layer density, $\rho_{\mathrm{NCL}}$, is affected by the solids composition and degree of compaction. Though the liquid fractions are generally over 50\%, compact nonconvective layers have smaller interstitial pores and thus somewhat less liquid. Also, if the interstitial liquid is highly concentrated, it will contribute to higher nonconvective layer density. The nonconvective layer densities range from $1300 \mathrm{~kg} / \mathrm{m}^{3}$ (lightly settled solids, dilute liquid) to $1800 \mathrm{~kg} / \mathrm{m}^{3}$ (compacted, concentrated liquid). 
When the densities are so different that the neutral buoyancy void fraction is fairly large (say in excess of 0.20 ), the potential exists for a large volume of gas to be stored in the nonconvective layer that could lead to a very large BDGRE. However, because the gas release rate increases with stored gas volume according to Eq. (2.6), it is highly unlikely that the void fraction can achieve the high value required. When the densities are so close together that the neutral buoyancy void fraction is small (say 0.05-0.10), much less gas accumulation is required for buoyancy, and the likelihood of a BDGRE can be high. This is particularly true because a highly concentrated liquid whose density approaches that of the nonconvective layer not only has a typically high gas generation rate but is also associated with a deep nonconvective layer, which reduces the release rate.

Finally, convective layer density also affects the average pressure at which gas is stored in the nonconvective layer. All other conditions being equal, the in situ pressure of retained gas increases linearly with $\rho_{\mathrm{CL}}$. Hence the potential gas release volume into the dome headspace also increases with $\rho_{\mathrm{CL}}$.

\subsubsection{Gas Retention Properties}

There is no single property or feature of the waste that can be evaluated to determine whether a tank can accumulate gas and how much gas will eventually be retained. By considering gas retention from a microscopic viewpoint, a group of properties are identified that influence gas retention just as in the above consideration of the overall, macroscopic balance of gas generation and release.

Bubbles, which compose the greatest volume of flammable gas in a tank, are the most important mode of gas retention concerning flammability. The principal mechanism of bubble retention in layers of settled solids is dominated by the waste strength and capillary forces; direct attachment of bubbles to particles plays a minor though necessary role, ${ }^{\text {(a) }}$ and gas volume that can be absorbed on solid surfaces is negligible. The two dominant retention mechanisms are discussed below.

Bubbles are held in the interstitial spaces or pores between particles by capillary forces when the interparticle lithostatic load is sufficient to hold the particles in contact against the force of the bubble's internal pressure trying to push them apart. These bubbles assume an irregular, dendritic shape, conforming to the passages between the particles. This retention mechanism requires either relatively large particles that allow larger bubbles with lower internal pressure, or a deep waste column that increases the lithostatic load, or both. If the internal bubble pressure is sufficient to push the surrounding particles apart, bubbles are retained by the yield strength of the waste.

Whether a bubble is held by waste strength or capillary force can be determined by a Bond number criterion developed by Gauglitz et al. (1994, 1995, 1996). It comprises a ratio of

(a) Bubbles must remain attached to "nursemaid" particles to grow from nucleation to a size $(50-100 \mu$ in diameter) at which their internal pressure is low enough to prevent the gas being driven back into solution (Peurrung et al. 1998). 
gravitational force to surface tension force and a ratio of waste strength force to surface tension force. If the Bond number exceeds unity, bubble retention is by capillary forces in the porefilling configuration. The Bond number is expressed as

$$
\mathrm{N}_{\mathrm{Bo}}=\frac{\Delta \rho g \mathrm{H}_{\mathrm{S}} \mathrm{D}_{\mathrm{p}}}{4 \sigma}+\frac{\tau_{\mathrm{y}} \mathrm{D}_{\mathrm{p}}}{4 \sigma}\left(\frac{\mathrm{A}_{2}}{\mathrm{~A}_{1}}\right)>1
$$

where

$$
\begin{aligned}
& \mathrm{H}_{\mathrm{S}}=\text { the height of the lithostatic column above the bubble } \\
& \mathrm{D}_{\mathrm{P}}=\text { the mean pore diameter through which a bubble must pass to escape } \\
& \text { retention. It is assumed to be represented by the mean particle diameter. } \\
& \Delta \rho=\text { the difference between solid and liquid density } \\
& \sigma \quad=\text { the surface tension } \\
& \tau_{\mathrm{Y}} \quad=\text { the yield stress } \\
& \mathrm{A}_{2} / \mathrm{A}_{1}=\text { a ratio related to how the yield stress resists bubble expansion; } \\
& \text { it was estimated at } 2.8 \text { by Gauglitz et al. (1995). }
\end{aligned}
$$

In the DST liquid-over-solid waste configuration, the solid particles in the nonconvective layer are immersed in liquid, which greatly reduces the lithostatic load by the resulting buoyant force, particularly in saltcake waste. The mean particle size is on the order of a micron. With such small particles, the Bond number defined by Eq. (2.7) is much less than unity through the entire depth of the nonconvective layer. Thus bubbles are able to displace the surrounding particles, and all significant gas retention in DSTs is due to waste strength.

The shape of a particle-displacing bubble is determined by a balance of surface tension and waste strength. Surface tension pulls relatively weak waste or small bubbles into an approximately spherical shape. If the effect of waste strength is greater than that of surface tension forces, the bubble grows into the weakest area of the waste surrounding it and assumes a dendritic shape. Particle-displacing bubbles that are not dendritic are called "round" bubbles, even though they may be ellipsoidal or similarly distorted from a truly spherical shape. A bubble shape criterion is derived by scaling the relative importance of strength to surface tension. The maximum diameter of round bubbles before they begin to assume a dendritic shape is given by

$$
\mathrm{D}_{\mathrm{b}}<\frac{\sigma}{\tau_{\mathrm{y}}}
$$

where $\tau_{y}$ is the yield stress, $\sigma$ is the surface tension, and $D_{b}$ is the bubble diameter. Eq. (2.8) suggests that bubbles in the lower half of the nonconvective layer have some dendritic character, while those in the upper portion are likely to be nearly spherical.

A bubble can grow only until its buoyancy exceeds the ability of the waste to hold it in place. A criterion for incipient motion of a sphere within material with a yield stress, $\tau_{\mathrm{y}}$, can be derived by a simple balance of surface force and buoyant force. The resulting limiting diameter is expressed as 


$$
\mathrm{D}_{\mathrm{b}}<\frac{6 \tau_{\mathrm{y}}}{\rho_{\mathrm{w}} \mathrm{g}}
$$

where $\rho_{\mathrm{W}}$ is the bulk waste density and $\mathrm{g}$ is the acceleration of gravity. Eq. (2.9) also gives the limiting height of dendritic particle-displacing bubbles. When the hydrostatic top-to-bottom pressure difference exceeds the material strength, the bottom of the bubble pinches off, and the top of the bubble pushes upward in a manner similar to, but much faster than, percolation of pore-filling bubbles. If the waste is moderately strong the bubbles do not collapse completely, and dendritic networks form that provide continuous gas release.

Considering the constraints of both Eq. (2.8) and (2.9) with typical DST nonconvective layer properties, the maximum diameter of a round bubble that can be retained is about $1 \mathrm{~cm}$. This size is consistent with observations in waste and simulants. A very large round bubble that could contain a hazardous amount of gas simply cannot exist. The volume average bubble size is about a millimeter, much larger than the solid particles surrounding it (Peurrung et al. 1998).

Based on the foregoing discussion and inspection of Eq. (2.7) through (2.9), the following list gives the physical parameters important for determining gas retention characteristics:

$$
\begin{aligned}
& H_{\mathrm{S}}=\text { thickness of the settled solids layer (nonconvective layer height) } \\
& \mathrm{D}_{\mathrm{P}}=\text { effective solid particle diameter, represents the size of pores between particles } \\
& \rho_{\mathrm{S}}=\text { density of solid particles } \\
& \rho_{\mathrm{L}}=\text { density of the supernatant (convective layer density) or interstitial liquid } \\
& \rho_{\mathrm{W}}=\text { bulk density of the waste (nonconvective layer bulk density) } \\
& \sigma=\text { surface tension } \\
& \tau_{\mathrm{y}}=\text { bulk waste yield stress. }
\end{aligned}
$$

Note the reappearance of the layer densities and nonconvective layer height, which have already been identified as important parameters from a macroscopic viewpoint. The nonconvective layer height also has a direct influence on the yield stress because it is approximately proportional to the lithostatic load and has been shown to vary almost linearly with depth (Meyer et al. 1997).

The yield stress is the one property that collects the influences of all the complexities of interparticle interaction. These might include particle shape, particle heterogeneity, wettability (contact angle of the liquid-gas-solid interface), interparticle forces, occurrence of chemical reactions that "glue" particles together, etc. However, because the bubbles are much larger than individual particles, the overall yield stress, which is the integrated result of all such factors, is the primary consideration. Even if they could be quantified, the specific mechanisms by which the yield stress assumes its value are not important in assessing gas retention once the yield stress is known.

Some differences can be noted in the properties for sludge and saltcake wastes listed above. Sludge tends to have somewhat smaller particles and a larger difference between solid and liquid densities. Though no in situ measurements have yet been made, the yield stress is expected to be somewhat less in sludge but should still increase with depth in the nonconvective layer. The 
surface tension in a sludge supernatant should be only slightly lower (closer to that of water) than the more concentrated liquid associated with saltcake.

Despite these differences, Eq. (2.7) through (2.9) indicate that there is little effective difference in gas retention behavior in DSTs between saltcake and sludge on the scale of an individual bubble. The bubble configuration in both wastes will be entirely particle-displacing bubbles that are several orders of magnitude larger than the particles that restrain them. The average bubble size should be somewhat less in sludge. A deep nonconvective layer in saltcake will tend to have dendritic bubbles near the bottom, but round bubbles should compose the largest fraction of the gas volume in both wastes.

\subsection{Susceptible Tanks}

The preceding discussion of the macroscopic and microscopic aspects of gas retention in DSTs clearly indicates only a few dominant conditions that exacerbate gas retention and increase the probability of BDGRE behavior:

- Deep convective layer (more than $2 \mathrm{~m}$ )

- Deep nonconvective layer (more than $2 \mathrm{~m}$ )

- Small neutral buoyancy void fraction (small difference between convective and nonconvective layer density)

- High gas generation rate.

Though sludge and saltcake wastes are both capable of retaining gas, all four primary gas retention conditions are usually found together only in saltcake tanks, and only five of these exhibit BDGRE behavior (six including pre-mitigation SY-101). ${ }^{\text {(a) }}$ In these tanks, concentration of the waste by evaporation brought the waste to BDGRE conditions.

\subsubsection{Tank Fill Histories}

Three major wastes are present in these tanks. Double-shell slurry (DSS) is the most concentrated form. It is considered a solid in tank inventories and is concentrated past the sodium aluminate saturation boundary in the evaporator. Double-shell slurry feed (DSSF) is somewhat less concentrated than DSS, required to be just under the sodium aluminate saturation boundary in the evaporator. Complexed concentrate (CC) is similar in composition to DSS and DSSF but contains more than $10 \mathrm{~g} / \mathrm{L}$ total organic carbon (TOC). All three wastes are below the sodium nitrate and sodium nitrite saturation boundary in the evaporator but become saturated and precipitates form on cooling.

SY-101 had the most concentrated waste of the DSTs and exhibited the largest gas releases by far (Hedengren et al. 2000). It was filled between 1977 and 1980 in a series of five evaporator campaigns and transfers consisting of DSS and CC, as listed in Table 2.1. Evaporation reduced the volume of the waste received by SY-101 by $25 \%$ to $30 \%$. The tank

(a) Three sludge tanks, AZ-101, AZ-102, and AY-102, have gas generation rates that exceed those of the five BDGRE tanks, and most DSTs have a deep layer of supernatant liquid. 
began having BDGREs shortly after filling was complete. The DSS waste supplied the concentrated salts that precipitated into a deep nonconvective layer, and the CC waste provided organic species that greatly increase the gas generation rate. Prior to concentration in the evaporator, none of the SY-101 evaporator feed wastes exhibited BDGRE behavior. These feeds were less concentrated than DSSF.

Table 2.1. SY-101 Fill Summary 1977 Through 1980

\begin{tabular}{|l|l|l|l|l|}
\hline $\begin{array}{c}\text { Campaign } \\
\text { Type }\end{array}$ & Waste Type & $\begin{array}{c}\text { Feed to } \\
\text { Evaporator (gal) }\end{array}$ & $\begin{array}{c}\text { Received in } \\
\text { SY-101 (gal) }\end{array}$ & $\begin{array}{c}\text { Volume Reduction } \\
\text { Factor }\end{array}$ \\
\hline Evaporator & DSS & 365,000 & 275,500 & 0.25 \\
\hline Evaporator & CC & 524,400 & 365,600 & 0.30 \\
\hline Transfer & CC & - & 133,300 & - \\
\hline Transfer & CC & - & 59,500 & - \\
\hline Evaporator & DSS & 313,000 & 231,300 & 0.26 \\
\hline
\end{tabular}

SY-103 also received DSS and CC but only to 200 inches (about half full). It did not begin to exhibit typical BDGRE behavior until an additional 60 inches of dilute liquid was added. Apparently, the additional supernatant liquid depth provided sufficient gas expansion to release gas when the waste became buoyant. AN-104, AN-105 and AW-101 consist mainly of DSSF and are filled almost to capacity. Each began BDGRE behavior, as indicated by level drops, shortly after filling.

AN-103 contains DSS over a rather deep, 2-m heel left after salt-well pumping had removed the liquid. The convective and nonconvective layer densities are higher even than those of $\mathrm{SY}-101$, but the radionuclide loading is less and the gas generation rate is correspondingly much lower. The surface level rose 17 inches in the first three years after filling and continued to rise until becoming steady in 1997, after reaching a total growth of 21 inches. The resulting void fraction in this tank is very close to neutral buoyancy, but GREs in this tank are very small and do not match the expectations of such a concentrated tank. Nevertheless, the tank currently contains the largest gas volume of the five (only SY-101 before mitigation contained a larger gas volume).

\subsubsection{Creating a Problem Tank}

The tanks are relatively well characterized in their current state. That is, those that exhibit BDGREs are identified and a sufficient number have been analyzed to determine the average release volume and variability. While the release behavior of these tanks is relatively benign, it is important to avoid making transfers or otherwise changing the waste configuration or properties in a tank that could cause BDGREs where none occurred before.

The same four conditions, deep convective layer, deep nonconvective layer, low neutral buoyancy void fraction, and high gas generation rate, apply to a new tank as well. Below are some examples of operations that could exacerbate gas retention and/or release: 
- Adding liquid to a tank with very little supernatant and a deep nonconvective layer: This could create a deep convective layer and provide sufficient energy to enable a BDGRE where none has occurred before.

- Adding slurry from a saltcake tank to a DST containing sludge with a high gas generation rate: Adding dissolved salt and salt particles would a) increase the depth of the nonconvective layer, $b$ ) decrease the neutral buoyancy void fraction, and c) increase the convective layer depth. All of these along with the existing high gas generation rate could satisfy all four conditions for BDGREs.

- Increasing the temperature (e.g., by reducing ventilation) of a saltcake DST with deep nonconvective and convective layers: Though heating would dissolve some solids and decrease the nonconvective layer height, increased temperature greatly increases the gas generation rate, possibly to the point at which BDGREs could occur.

- Adding slurry from a saltcake tank to another partially filled saltcake tank: Solids settling out of the slurry plus increased waste volume would a) increase the nonconvective layer depth, $b$ ) increase the convective layer depth, and c) increase the gas generation rate due to higher nonconvective layer temperature caused by increased depth. All these effects together could cause gas accumulation to the point of BDGREs.

There is a historical example of the last scenario. The supernate from SX-104 was pumped into the DST SY-103 in 1988. Prior to this addition, SY-103 exhibited slow level drops of 1 to 2 inches several times a year. After the addition from SX-104, however, sudden level drops of $\sim 2$ inches began, which indicate that the BDGREs persist to this day (Hedengren et al. 2000). Based on its waste level history, occasional BDGREs apparently occurred in SX-104 prior to removal of its supernate. None have been observed there since. 


\subsection{Parametric Indicators}

In the previous section, the basic underlying physics of the BDGRE process was described. In addition to general understanding, there is a need to be able to identify specific tanks or specific operations that alter the waste conditions in tanks that have the potential for BDGREs based on measurable or predictable waste parameters. Hence specific criteria or parametric indicators are needed. Various efforts to discover such indicators of tank behavior have been attempted over the years. All of the approaches have fallen into one of three categories: chemistry-based indicators, empirical indicators, and physically based indicators. Each type of indicator and its merits are addressed in separate sections below

\subsection{Chemistry-Based Indicators}

The idea behind chemistry-based indicators is that only tanks with certain chemical signatures will exhibit BDGREs. An expert panel review (Slezak et al. 1998) included three empirical chemical criteria proposed by Steve Agnew for screening tanks for BDGRE behavior:

- Sodium (Na) concentration above a specified value (10 molar), and TOC above a specified value ( 0.3 weight percent).

- Aluminum (Al) concentration above a specified value (0.9 molar), and TOC above a specified value ( 0.3 weight percent).

- Nitrite $\left(\mathrm{NO}_{2}{ }^{-}\right)$concentration above a specified value (2.0 molar), and TOC above a specified value ( 0.3 weight percent).

These chemistry-based criteria, while adequately indicating BDGRE behavior in some DSTs, fail to provide a clear delineation when applied broadly to all DSTs. ${ }^{\text {(a) }}$ Additionally, we know that the underlying physics of BDGREs is based in mechanics and transport phenomena whose behavior is governed by macroscopic properties. Chemistry plays a significant role in determining macroscopic properties. Because high sodium and nitrite concentrations cause additional solids precipitation and raise the liquid density, they could be considered surrogates for the nonconvective layer depth and the difference between the convective and nonconvective layer densities. Similarly, TOC, nitrite, and aluminum concentrations might loosely represent the gas generation rate (Hu 1999). However, beyond establishing basic trends, the chemical factors behind the properties are secondary to the properties themselves. One could say that chemistry sets the stage, but physics performs the play. Because of these factors, chemistrybased indicators are not recommended for predicting BDGRE behavior.

\subsection{Empirically Based Indicators}

There have been several attempts over the years to find empirical relationships that correlate groupings of physical parameters with BDGRE behavior (Estey and Guthrie 1996). The single

(a) CHG Memo 79000-00-031 from SST Closure Project to SY-101 Surface Level Rise Remediation Project File, June 19, 2000. Subject: White Paper, "Plan for Remediation of Gas Retention in SY-101." 
historical waste property found to correlate most closely with BDGRE behavior was tank average specific gravity, or density. ${ }^{\text {(a) }}$ A criterion for waste transfers was established based on the weighted mean of transferred waste and the waste in the receiver tank (Fowler 1995). ${ }^{\text {(b) }}$ If the weighted mean specific gravity was found by calculation to be in excess of 1.41, BDGRE behavior was considered a possibility and further analysis was required. Transfers are conducted such that the mean specific gravity remains less than the 1.41 limit.

A recognition that there was little physical basis for density alone to determine gas retention characteristics leading to BDGREs led to additional efforts to correlate groupings of parameters with BDGRE behavior. Estey and Guthrie (1996) tried several combinations of nonconvective layer and convective layer depth and specific gravity. The product of nonconvective layer depth and convective layer specific gravity was found to provide the clearest separation between tanks that exhibited BDGREs and those that did not. A criterion was developed based on this separation. If the product of nonconvective layer depth (in inches) and convective layer specific gravity exceeds 150 inches, BDGREs may occur.

\subsection{Physically Based Indicators}

A physically based model referred to as the buoyancy model has been developed from the theory of bubble transport (Meyer and Wells 2000). This model was developed to predict whether sufficient gas could build up in the nonconvective layer to make it buoyant, thereby initiating a BDGRE. Based on the balance between internal gas generation and slow gas release at the top, expressed in principle by Eq. (2.6), the model predicts a steady-state void fraction profile. If the integrated average void fraction is less than the neutral buoyant void fraction, a buoyant displacement cannot occur. On the other hand, an average void fraction greater than the neutral buoyant void fraction implies that a buoyant displacement would have occurred at some point prior to reaching the steady state.

In general, void growth due to gas generation is believed to occur by a combination of two effects, new bubbles nucleating from gas coming out of solution and existing bubbles growing through diffusion of dissolved gas to the bubble surfaces. Bounding solutions for steady-state void fraction are found from the general theory for two limiting cases. The first case assumes that the nucleation of new bubbles is uniform within the nonconvective layer, implying void growth occurs via new bubble development. The second case assumes that nucleation occurs only in a thin layer at the bottom of the layer. In the bulk of the nonconvective layer, gas generation only causes existing bubbles to grow, and no new ones are nucleated. The second case is consistent with the theory that nucleation is suppressed by diffusion of dissolved gas into existing bubbles. However, its predictions include non-BDGRE tanks with those that are observed to exhibit them. For this reason, the first case is recommended as the best discriminator.

(a) Reynolds DA. 1994. Evaluation of Specific Gravity versus Gas Retention. Internal memo 7E31094-024. Westinghouse Hanford Company, Richland, Washington.

(b) The weighted mean specific gravity includes both the convective and nonconvective layer specific gravity and that of the transferred waste. 
The ratio of average steady state void fraction to neutral buoyant void fraction for the case of constant nucleation is

$$
\mathrm{BR}=\frac{\alpha_{\mathrm{NCL}}}{\alpha_{\mathrm{NB}}}=\frac{\mathrm{C}}{\rho_{\mathrm{NCL}}-\rho_{\mathrm{CL}}}\left(\frac{\mathrm{gT}}{\mathrm{p}}\right)^{1 / 3} \mathrm{H}_{\mathrm{NCL}}^{2}
$$

where $\mathrm{T}$ and $\mathrm{p}$ are the average temperature $(\mathrm{K})$ and hydrostatic pressure $(\mathrm{Pa})$ in the nonconvective layer, respectively; $g$ is the molar gas generation rate per unit volume (moles $/ \mathrm{m}^{3}$ day); and $\mathrm{H}_{\mathrm{NCL}}$ is the nonconvective layer depth. The leading coefficient $C$ is adjusted so that the minimum ratio of average void fraction to neutral buoyancy void fraction predicted for the five tanks exhibiting BDGREs is exactly one. Using the most current data, AW-101 has the minimum buoyancy ratio, which is made equal to unity by setting $\mathrm{C}=866$.

Equation (3.1) is referred to as the buoyancy ratio, $B R$. If the buoyancy ratio is less than unity, then the model suggests that a BD is not possible even in steady state. However, if the ratio exceeds unity, the model suggests that periodic buoyant displacements will occur, preventing void buildup to a steady state.

It is more correct, considering the influence of the presence of gas on the nonconvective layer, to use the gas generation rate and nonconvective layer depth at zero void fraction, $\mathrm{g}_{0}$ and $\mathrm{H}_{0}$, respectively, in Eq. (3.1). These quantities are related to the steady-state values, $g$ and $\mathrm{H}_{\mathrm{NCL}}$, that are measured in existing tanks by the following:

$$
\mathrm{g}_{0}=\frac{\mathrm{g}}{1-\alpha_{\mathrm{NCL}}}
$$

and

$$
\mathrm{H}_{0}=\mathrm{H}_{\mathrm{NCL}}\left(1-\alpha_{\mathrm{NCL}}\right)
$$

If the void fraction is not known or a "new" tank is being evaluated that has not reached steady state, it is conservative to assume that $\alpha_{\mathrm{NCL}}=0$ and to use the measured quantities directly in Eq. (3.1). Using the zero-void quantities in Eq. (3.1) makes AN-103 the tank with the minimum buoyancy ratio which is set to unity by making $\mathrm{C}=973$.

\subsection{Data Requirements}

The three parametric indicators introduced above require various waste properties and parameters as inputs. Table 3.1 summarizes the parametric indicators and shows the inputs required for each. Note that, although the nonconvective layer average pressure appears explicitly in Eq. (3.1), it is determined by the weight of the waste above it. Hence, the nonconvective layer pressure is a function of convective and nonconvective layer densities and depths.

Data are available to evaluate existing tanks of concern. Many have been sampled recently, and several of the DSTs have had in situ rheology and void fraction measurements (Hedengren et 
al. 2000). The layer densities based on core sample or grab sample analyses are available in the Best Basis Inventory on the TWINS3 database or from tank characterization reports. The overall waste level and temperature are monitored continuously and recorded on the TMACS system retrievable via the TWINS3 database or PCSACS system. The crust (if any), convective layer, and nonconvective layer thicknesses can be inferred from the temperature profile, preferably from validation probe data if the tank has a multifunction instrument tree (MIT) installed (Meyer et al. 1997). Neutron and gamma logs are also available in tanks with MITs to provide additional confirmation of waste configuration (Hedengren et al. 2000; Rassat et al. 2000; Mahoney et al. 2000).

Table 3.1. Inputs Required for Parametric Indicators

\begin{tabular}{|l|c|c|c|c|c|c|}
\hline Criterion & $\begin{array}{c}\text { NCL } \\
\text { Depth }\end{array}$ & $\begin{array}{c}\text { CL } \\
\text { Depth }\end{array}$ & $\begin{array}{c}\text { NCL } \\
\text { Density }\end{array}$ & $\begin{array}{c}\text { CL } \\
\text { Density }\end{array}$ & $\begin{array}{c}\text { Gas Gen. } \\
\text { Rate }\end{array}$ & $\begin{array}{c}\text { NCL } \\
\text { Temp }\end{array}$ \\
\hline$\rho_{\mathrm{AVE}}<1.41$ & $\mathrm{x}$ & $\mathrm{x}$ & $\mathrm{x}$ & $\mathrm{x}$ & & \\
\hline $\begin{array}{l}\mathrm{H}_{\mathrm{NCL}} \mathrm{x} \mathrm{SpG}_{\mathrm{CL}} \\
<150 \text { in. }\end{array}$ & $\mathrm{x}$ & & & $\mathrm{x}$ & & \\
\hline $\mathrm{BR}<1$ & $\mathrm{x}$ & $\mathrm{x}$ & $\mathrm{x}$ & $\mathrm{x}$ & $\mathrm{x}$ & $\mathrm{x}$ \\
\hline
\end{tabular}

The gas generation rate can be estimated from the waste chemical composition and temperature using the empirical correlation of $\mathrm{Hu}$ (1999) or computed directly from the measured gas release history using several years of headspace gas monitoring data (Hedengren et al. 2000). The uncertainty in either the correlation or the calculation results is relatively high. However, this quantity is only used in the buoyancy ratio, and because it is raised to the $1 / 3$ power, a relatively large uncertainty can be tolerated.

In the case of a planned future waste transfer or other waste-altering operation, the ultimate steady-state tank conditions must be predicted to evaluate the parametric indicators. The predictions must include the effects of dissolution and precipitation when the wastes are mixed and/or diluted. Of particular importance is the change in waste temperature. The types of waste configuration expected and issues involved in predicting the resulting steady state are discussed in Section 4. 


\subsection{Applying Parametric Indicators}

The parametric indicators historically were developed out of efforts to understand BDGRE phenomena and provide a means to distinguish BDGRE and non-BDGRE tanks. BDGRE behavior in existing DSTs has provided a means of calibrating and validating the indicators. As the Hanford Site transitions from a mode of safe storage and monitoring to that of waste feed delivery to vitrification plants, it is important to avoid creating new waste tank configurations by waste transfer and mixing operations that have the potential for BDGREs. The parametric indicators provide a tool to help guide waste transfer strategies to ensure safe outcomes.

This section discusses some of the issues in applying the parametric indicators to plan and evaluate the results of various tank waste operations. Section 4.1 describes potential operations that change the waste configuration, including waste mixing, removal, addition, and dilution, in general terms. Section 4.2 identifies important effects that must be considered in predicting the end state of the tank in order to apply the parametric indicators in advance. Finally, Section 4.3 gives examples and results of using the parametric indicators for transfer and dilution of SY-101 and sluicing C-106 waste into AY-102.

\subsection{Creating New Waste Configurations}

New waste configurations are created from existing ones. By existing waste configurations, we are referring to conditions in a tank that has been relatively undisturbed for an extended period of time except, for example, periodic water additions or water loss through evaporation and other minor waste-disturbing activities such as core sampling. These tanks generally have a distinct and stable nonconvective layer that has reached steady-state compaction and in which the soluble solids and solution are in equilibrium. The waste surface level and temperature are stable except for minor seasonal variations. The five tanks that exhibit small, random BDGREs have reached a different kind of steady state where parts of the waste are at different stages of gas retention but the tank average is essentially stationary. The small BDGREs are very local and are considered a minor waste disturbance.

This section describes common operations that alter the waste configuration and how the change occurs. Three basic categories of operations are considered: waste modification (i.e., without adding or removing material), waste removal, and waste addition. In reality, most operations include all three, but the overall effects are more or less additive.

\subsubsection{Waste Modification}

The most common and most important waste modification is a temperature change that accompanies changes in headspace or annulus ventilation flows. A change in temperature affects all aspects of BDGRE behavior, as will be discussed in more detail in Section 4.2. By way of example, however, four of the five DSTs exhibiting BDGREs have experienced measurable cooling $\left(5-10^{\circ} \mathrm{F}\right.$ in average temperature) over the past $4-5$ years. No significant change in nonconvective layer depth was observed as a result of this cooling; however, the tanks' gas release behavior changed considerably with much smaller but more frequent releases. During 
the transition period of 12-18 months in 1996-1997, the tanks had few releases (AW-101 had none). In these tanks, temperature had a powerful effect on BDGRE behavior.

Mixing is the other waste modification that is expected to be common during waste feed delivery. The feed tanks and interim storage tanks are all slated to have mixer pumps installed. SY-101 was mixed from December 1993 to April 2000 to prevent gas retention. While the waste is actually mixed and most of the solids are suspended, gas retention is negligible and BDGREs are not a concern. However, when mixing ceases, solids eventually settle out into a nonconvective layer that can potentially retain gas.

\subsubsection{Waste Removal}

Waste removal includes removal of mixed slurry following mixer pump operation, decanting convective layer liquid, or direct pumping of nonconvective layer material. If a stratified tank has been mixed prior to a waste transfer, as was the case for SY-101, then both solids and liquids will be removed from the tank. After a period of settling, the tank will return to a stratified state, only now with reduced convective layer and nonconvective layer depths in approximately the original proportion. Both the layer densities and gas generation rate per unit volume would remain the same as the pretransfer values if the waste stayed at the original temperature. However, the waste will cool due to the increased surface-to-volume ratio after waste removal. Besides the cooling effect, BDGREs would be much less a hazard after mixed waste removal because the increased headspace dilutes releases and hydrostatic pressure and nonconvective layer thickness are reduced.

Decanting supernatant liquid may be used as one step in delivering waste from the DSTs to the vitrification plant. Depending on the amount of supernatant liquid removed, BDGREs may be impossible after decanting. If less than about two meters of liquid remains, there is probably not enough potential energy to cause a gas release. The energy calculation described by Meyer and Wells (2000) or by Meyer et al. (1997) should be applied. If sufficient supernatant liquid remains to make BDGREs possible, cooling effects again need to be accommodated in estimating the steady-state waste condition.

Decanting will cause a pressure reduction that will likely produce several BDGREs in tanks that already exhibit them spontaneously. As the hydrostatic pressure falls during decanting, the gas in the nonconvective layer expands proportionally. Assuming the nonconvective layer contains a number of gobs in progressive stages of gas retention, more than half of them will expand to buoyancy during the progress of decanting. ${ }^{\text {(a) }}$ The same effect might cause a tank not currently experiencing BDGREs to do so. However, to predict whether this would occur, the current average nonconvective layer void fraction must be estimated.

Direct removal of nonconvective layer material from under the supernatant liquid in an unmixed tank is technically possible if the strength is low. However, this operation has not yet been proposed and is not considered further.

(a) Stewart CW, PA Meyer, and BE Wells. 1999. Potential for Inducing Gas Releases in Double-Shell Tanks During Retrieval. PNNL letter report TWS99.44 Rev. 1, August 1999. 


\subsubsection{Waste Additions and Dilution}

When the waste in the receiver tank has been mixed just before, during, or after the transfer, the incoming waste can be considered mixed with it and composition determined approximately by volume averaging. If the receiver tank is not mixed in conjunction with the incoming transfer, the waste will stratify. The incoming solids will settle out onto the existing nonconvective layer, and the liquids will stratify according to density. The stratified liquid layers will eventually mix by diffusion and co-convection, but the two nonconvective layers are essentially permanent. The effects of a temperature increase due to decreased surface-to-volume ratio need to be considered in predicting the final steady state.

Waste removal and back-dilution with water can reduce or eliminate the risk of BDGRE in a tank with soluble waste. ${ }^{(a)}$ Dilution dissolves solids and directly reduces the height of the nonconvective layer. The concentration of gas generating species is also reduced, which causes the gas generation rate to decrease. If the amount of dilution is large enough to dissolve all of the solids with the highest solubilities, the density will fall. This increases the neutral buoyancy void fraction, thereby requiring additional gas retention before a BDGRE can occur. As in most other cases, the effects of a temperature decrease due to reduced heat load per unit volume must be considered. Mixing is required after diluent is added to make all this happen efficiently. The exception would be diluting to dissolve a crust layer where the density gradients work to keep solids in contact with the most dilute liquid. In the absence of post-dilution mixing, subsurface water addition can aid in the mixing process.

If adequate mixing is not performed with the dilution, dissolution will be very slow. Because water is lighter than the convective liquid, it will form a layer on top of the waste until diffusion eventually brings it into equilibrium with the rest of the liquid. This process could take a significant amount of time. Further diffusion of the slowly diluting convective layer into the nonconvective layer could take years.

\subsection{Important Issues in Predicting the Waste Configuration}

The discussion of waste modification in Section 4.1 has introduced several important effects that will be considered here in more detail. The most important are temperature changes and time for the waste to reach the new configuration.

\subsubsection{Temperature Effects}

Reducing the waste volume increases its surface-to-volume ratio. This increases heat dissipation relative to generation and lowers the average waste temperature. The average temperature in the nonconvective layer would decrease even farther because its temperature difference is proportional to the square of HNCL. However, in a concentrated saltcake tank, cooling would cause precipitation that would increase HNCL that would counteract some of the effects of the transfer. Precipitation increases the convective and nonconvective layer density

(a) Waste removal is usually required to make room for the diluent but is not absolutely necessary. 
difference and increases the neutral buoyancy void fraction. Cooling reduces the gas generation rate by a large factor because it is extremely sensitive to temperature. This is probably the most important and beneficial effect of cooling, as evidenced by the behavior of the five DSTs discussed in Section 4.1.

The reverse of all these effects occurs when the waste volume increases as surface-to-volume ratio decreases and temperature rises. While the resultant heating dissolves solids whose solubilities increase with temperature, the gas generation increases and BDGREs would be expected to increase in size and frequency. However, additions to a tank are likely to be more dilute than the existing waste, which counteracts the effect of decreasing surface-to-volume ratio.

The combined effects of these complex coupled processes can be quantified only by laboratory tests of dilution and/or mixing of different wastes at several temperatures covering the expected range. Application of a detailed thermal model is also necessary to bracket the expected thermal state of the tank, including any changes to ventilation rates. With these data the important properties at the end state can be predicted with reasonable accuracy.

\subsubsection{Time for Steady State}

Whether BDGREs can occur may not be as important as when they might begin. This is controlled by several processes that progress very slowly, so the ultimate steady state, in terms of gas retention, requires several years to develop.

To retain gas, a nonconvective layer must develop and gain sufficient strength to retain gas. Settling of the micron- or submicron-sized particles is very slow. In AY-102, initial settling after a batch of sludge was sluiced in from C-106 required on the order of a week, though the nonconvective layer height continued to decrease for several more weeks. Initial settling in SY-101 after mixing was terminated on April 2, 2000 occurred over about six weeks, with compaction continuing for several months longer.

Assuming a nonconvective layer, gas retention is an even slower process. At typical gas generation rates, at least 2-3 years are required to achieve buoyancy from a degassed state, even at $100 \%$ retention. Gas retention from a degassed state also causes the waste level to increase. If the neutral buoyancy void fraction is $10 \%$, a 100 -inch nonconvective layer with an average pressure of 2 atm would have to expand by 6 inches to reach buoyancy. This large level rise is easily detectable, allowing action to prevent a BDGRE to be taken well before one becomes imminent. Neither of the "new" tanks (AY-102 or SY-101) nor any of the existing non-watchlist tanks show level growth that would indicate an eventual approach to BDGREs.

\subsection{Example Applications}

One or all of the parametric indicators have been used in planning for two major operations: transfer and back-dilution of SY-101 and sluicing of C-106 sludge into AY-102. The transfer and back-dilution in SY-101 was planned based on the three physical parametric indicators. The physical properties and waste configuration after transfer and dilution were estimated based on an analysis of the dilution test data from 1991 and 1998 core samples and conservation of mass, assuming the volume fraction of settled solids after dilution would return to original value 
(Rassat et al. 2000). The uncertainties of the predicted physical parameter values were determined with a detailed Monte Carlo simulation on the transfer and back-dilution model. The gas generation rate was estimated conservatively initially by reducing the historic generation rate by the planned dilution ratio. Later, a more realistic estimate was made using the Hu (1999) correlation and the post-dilution composition determined from a grab sample. The parametric indicators showed that a dilution ratio approaching 1:1 (water to original waste volume) was necessary to preclude BDGREs. The actual dilution ratio was 0.8:1.

The buoyancy ratio was used to assess whether the combined wastes in AY-102 after transfer of waste from C-106, with correspondingly deeper nonconvective layer, could have BDGREs. Predictions of waste conditions were based on results of laboratory waste mixing tests. However, because the sluicing operation required almost a year with long breaks between batches, the early data were available to confirm or adjust the initial predictions and assumptions. A major uncertainty was the "fluffing" factor, which is the ratio of the height of newly transferred solids a short time after initial settling to the original height of the same material in the donor tank. The lab results implied a "fluffing" factor about 1.4. Early post-sluicing data, however, showed no fluffing. The gas generation in AY-102 was initially assumed to be the sum of the pre-transfer rates in the two tanks. Later, however, we learned that a significant volume of gas generating material remained in $\mathrm{C}-106$, reducing the rate in AY-102. The buoyancy ratio for both predicted and actual post-sluicing conditions was less than one.

The predicted conditions in both SY-101 and AY-102 have proved to be reasonable estimates of what actually occurred, and the negative predictions of the BDGRE parametric indicators have been substantiated. Using the buoyancy ratio in AY-102 and all three physical indicators in SY-101, both tanks were predicted not to retain sufficient gas for BDGREs to occur. Based on current monitoring data, neither tank has shown any detectable gas retention.

The parametric indicators have been applied to all 28 DSTs. The buoyancy ratio (using zerovoid values), SpGCL*HNCL parameter, and average specific gravity are shown in Figures 4.1, 4.2 and 4.3 , respectively. The results indicate that 23 of the tanks have no BDGRE problem at all.

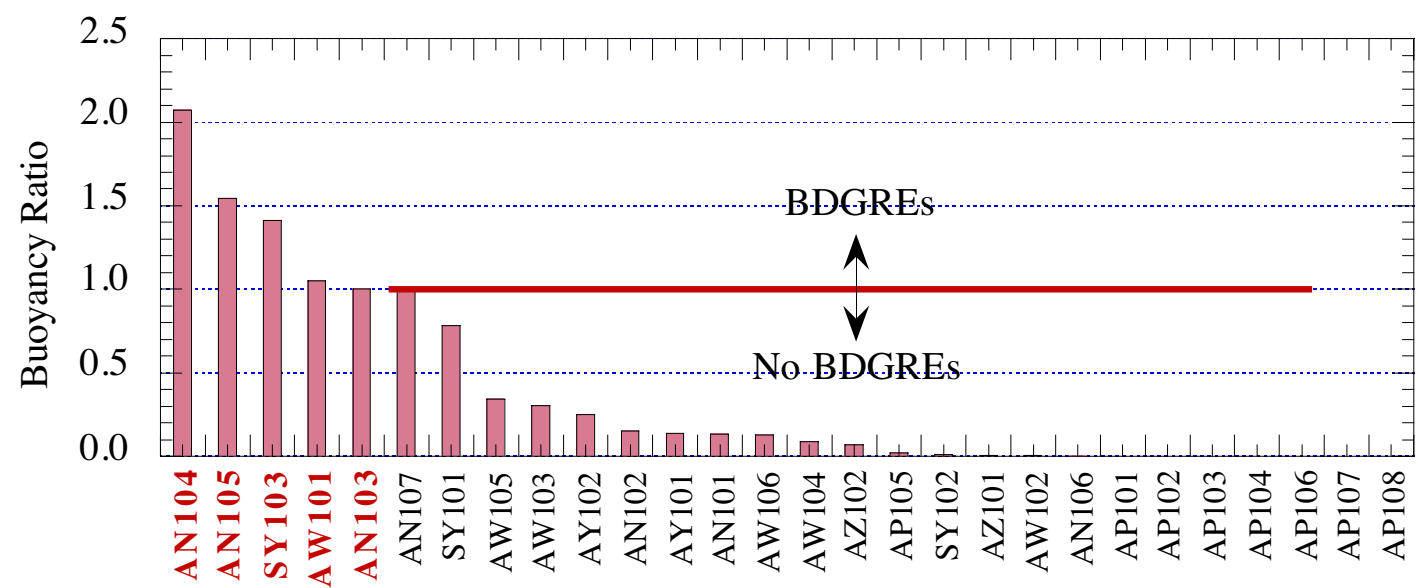

Figure 4.1. Buoyancy Ratio Parameter Values for all Hanford Double-Shell Tanks 


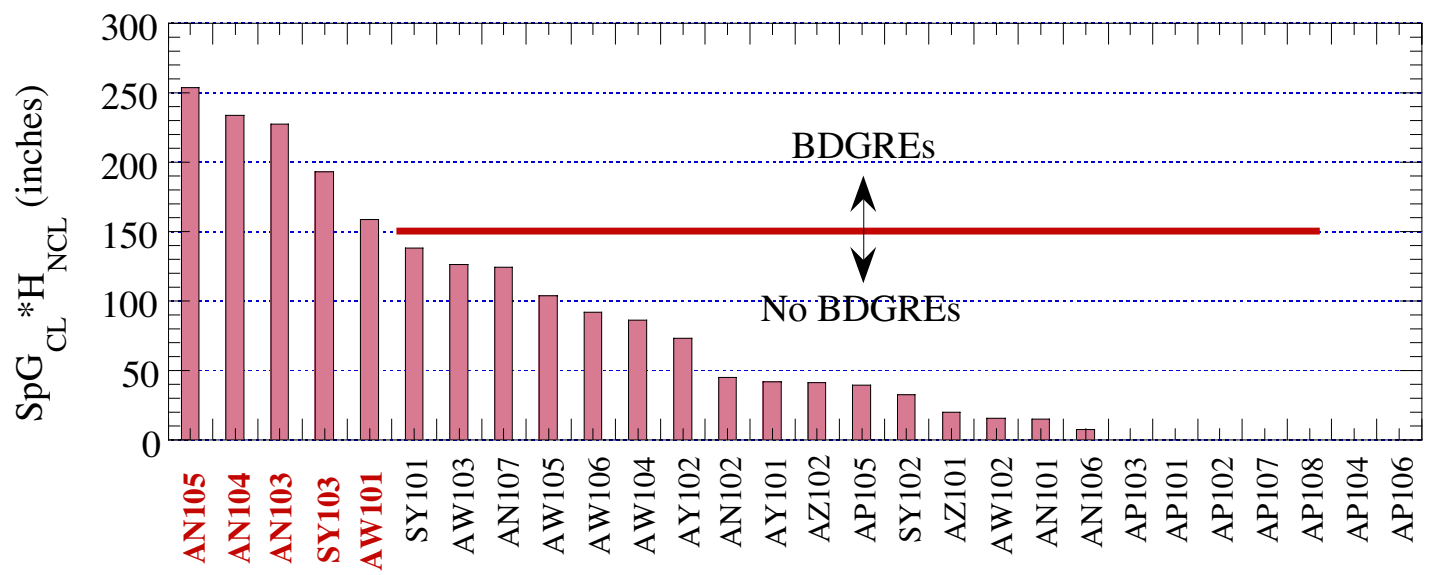

Figure 4.2. SpGCL*HNCL Parameter Values for all Hanford Double-Shell Tanks

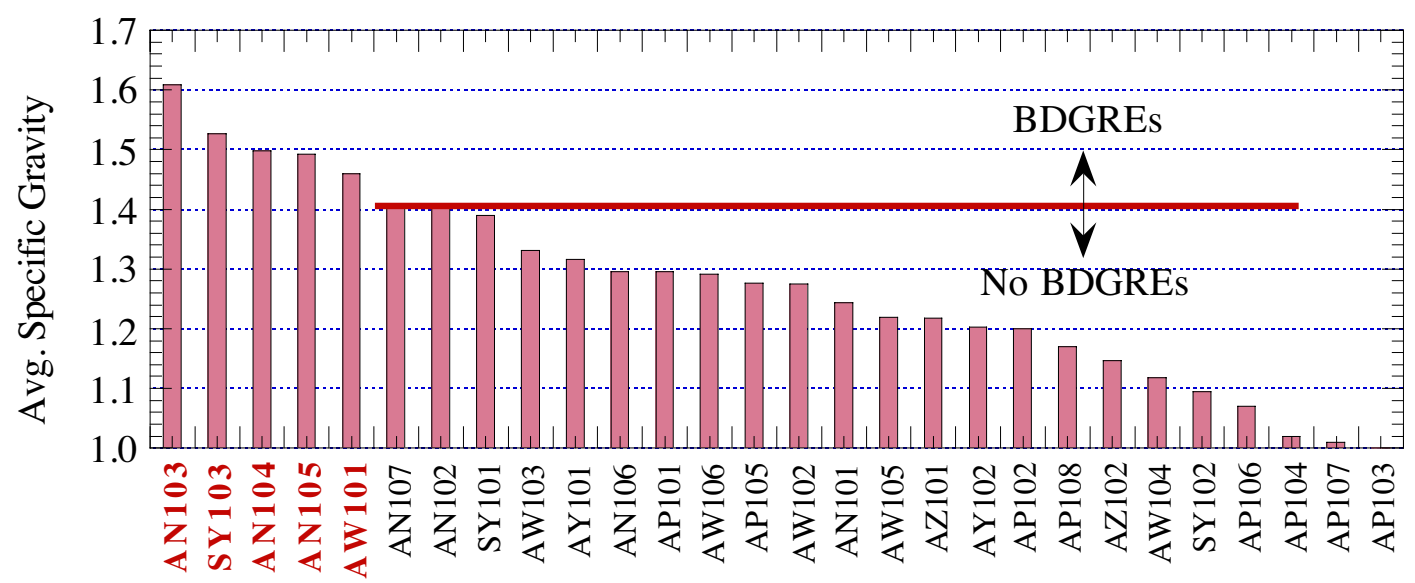

Figure 4.3. Average Specific Gravity for all Hanford Double-Shell Tanks

The indicators also show that only five of the tanks, AN-103, AN-104, AN-105, AW-101, and SY-103, have BDGREs. These results are consistent with the current behavior of the DSTs. Also shown in the three figures are results for SY-101 as of August 31, 2000. According to the parametric indicators, BDGRE behavior in SY-101 has been mitigated. 


\subsection{References}

Allemann RT, TM Burke, DA Reynolds, and DE Simpson. 1993. Assessment of Gas Accumulation and Retention-Tank 241-SY-101. WHC-EP-0576, Westinghouse Hanford Company, Richland, Washington.

Allemann RT, ZI Antoniak, WD Chvala, LE Efferding, JG Fadeff, JR Friley, WB Gregory, JD Hudson, JJ Irwin, NW Kirch, TE Michener, FE Panisko, CW Stewart, and BM Wise. 1994. Mitigation of Tank 241-SY-101 by Pump Mixing: Results of Testing Phases $A$ and $B$. PNL-9423, Pacific Northwest Laboratory, Richland, Washington.

Brewster ME, NB Gallagher, JD Hudson, and CW Stewart. 1995. The Behavior, Quantity, and Location of Undissolved Gas in Tank 241-SY-101. PNL-10681, Pacific Northwest Laboratory, Richland, Washington.

Estey SD and MD Guthrie. 1996. An Analysis of Parameters Describing Gas Retention and Release Behavior in Double-Shell Tank Waste. WHC-SD-WM-TI-755, Westinghouse Hanford Company, Richland, Washington.

Fowler KD. 1995. Tank Farm Waste Compatibility Program. WHC-SD-WM-OSD-015 REV. 1, Westinghouse Hanford Company, Richland, Washington.

Gauglitz PA, LA Mahoney, DP Mendoza, and MC Miller. 1994. Mechanisms of Gas Bubble Retention. PNL-10120, Pacific Northwest National Laboratory, Richland, Washington.

Gauglitz PA, SD Rassat, MR Powell, RR Shah, and LA Mahoney. 1995. Gas Bubble Retention and Its Effects on Waste Properties: Retention Mechanisms, Viscosity, and Tensile and Shear Strengths. PNL-10740, Pacific Northwest National Laboratory, Richland, Washington.

Gauglitz PA, SD Rassat, PR Bredt, JH Konynenbelt, JM Tingey, and DP Mendoza. 1996. Mechanisms of Gas Bubble Retention and Release: Results for Hanford Waste Tanks 241-S-102 and 241-SY-103 and Single-Shell Tank Simulants. PNNL-11298, Pacific Northwest National Laboratory, Richland, Washington.

Hanlon BM. 2000. Waste Tank Summary Report for the Month Ending January 31, 2000. HNF-EP-0182-142, CH2MHILL Hanford Group, Inc., Richland, Washington.

Hedengren DC, KM Hodgson, WB Barton, CW Stewart, JM Cuta, and BE Wells. 2000. Data Observations on Double-Shell Flammable Gas Watch List Tank Behavior. RPP-6655, CH2M Hill Hanford Group, Inc., Richland, Washington.

Hu TA. 1999. Empirical Rate Equation Model and Rate Calculations of Hydrogen Generation for Hanford Tank Waste. HNF-3851 Rev. 0, Lockheed Martin Hanford Corporation, Richland, Washington.

Mahoney LA, WB Barton, JM Conner, NW Kirch, CW Stewart, BE Wells. 2000. Results of Waste Transfer and Back-Dilution in Tanks 241-SY-101 and 241-SY-102. PNNL-13267, Pacific Northwest National Laboratory, Richland, Washington.

Meyer PA, ME Brewster, SA Bryan, G Chen, LR Pederson, CW Stewart, and G Terrones. 1997. Gas Retention and Release Behavior in Hanford Double-Shell Waste Tanks. PNNL-11536 Rev.1, Pacific Northwest National Laboratory, Richland, Washington. 
Meyer PA and BE Wells. February 2000. "Understanding Gas Release Events in Hanford Double-Shell Tanks." Proceedings of Waste Management 2000, Tucson, Arizona.

Peurrung LM, LA Mahoney, CW Stewart, PA Gauglitz, LR Pederson, SA Bryan, and CL Shepard. 1998. Flammable Gas Issues in Double-Contained Receiver Tanks. PNNL-11836, Pacific Northwest National Laboratory, Richland, Washington.

Rassat SD, CW Stewart, BE Wells, JM Cuta, WL Kuhn, ZI Antoniak, KP Recknagle, G Terrones, JH Sukamto, VV Viswanathan, and DP Mendoza. 2000. Dynamics of Crust Dissolution and Gas Release in Tank 241-SY-101. PNNL-13112, Pacific Northwest National Laboratory, Richland, Washington.

Slezak SE, DC Williams, W Cheng, F Gelbard, and DR Bratzel. 1998. Refined Safety Analysis Methodology for Flammable Gas Risk Assessment in the Hanford Site Tanks. HNF-SD-WMES-410 Rev. 2, Fluor Daniel Hanford, Inc., Richland, Washington.

Stewart CW, JD Hudson, JR Friley, FE Panisko, ZI Antoniak, JJ Irwin, JG Fadeff, LE Efferding, TE Michener, NW Kirch, and DA Reynolds. 1994. Mitigation of Tank 241-SY-101 by Pump Mixing: Results of Full-Scale Testing. PNL-9959, Pacific Northwest Laboratory, Richland, Washington.

Stewart CW, JM Alzheimer, ME Brewster, G Chen, RE Mendoza, HC Reid, CL Shepard, and G Terrones. 1996. In Situ Rheology and Gas Volume in Hanford Double-Shell Waste Tanks. PNNL-11296, Pacific Northwest National Laboratory, Richland, Washington. 